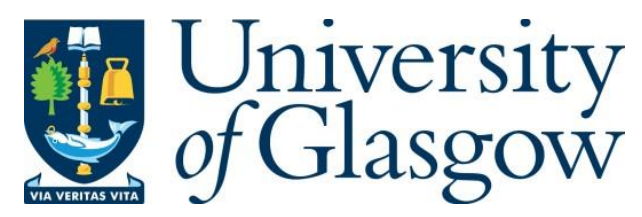

Fischer, M. P. et al. (2017) Germanium Nanoantennas for Plasmon-enhanced Third Harmonic Generation in the Mid Infrared. In: CLEO/Europe - EQEC 2017, Munich, Germany, 25-29 Jun 2017, ISBN 9781509067367.

There may be differences between this version and the published version. You are advised to consult the publisher's version if you wish to cite from it.

http://eprints.gla.ac.uk/152537/

Deposited on: 27 November 2017

Enlighten - Research publications by members of the University of Glasgow http://eprints.gla.ac.uk 


\title{
Germanium Nanoantennas for Plasmon-Enhanced Third Harmonic Generation in the Mid Infrared
}

\author{
Marco P. Fischer1, Aaron Riede1, Alexander Grupp1, Kevin Gallacher², Jacopo Frigerio³, \\ Giovanni Pellegrini ${ }^{4}$, Michele Ortolani ${ }^{5}$, Douglas J. Paul ${ }^{2}$, Giovanni Isella ${ }^{3}$, \\ Alfred Leitenstorfer ${ }^{1}$, Paolo Biagioni ${ }^{4}$, and Daniele Brida ${ }^{1}$ \\ ${ }^{1}$ Department of Physics and Center for Applied Photonics, University of Konstanz, 78457 Konstanz, Germany \\ 2 School of Engineering, University of Glasgow, Rankine Building, Oakfield Avenue, Glasgow, G12 8LT, UK \\ ${ }^{3}$ L-NESS, Dipartimento di Fisica del Politecnico di Milano, Via Anzani 42, 22100 Como, Italy \\ ${ }^{4}$ Dipartimento di Fisica, Politecnico di Milano, Piazza Leonardo da Vinci 32, 20133 Milano, Italy \\ ${ }^{5}$ Department of Physics, Sapienza University of Rome, 00185 Rome, Italy
}

Recent advances in semiconductor film deposition allow for the growth of heavily-doped germanium with effective plasma frequencies above $60 \mathrm{THz}$, corresponding to wavelengths below $5 \mu \mathrm{m}$. This technology paves the way for mid-infrared nanoplasmonics with application in integrated telecommunication systems and enhanced molecular sensing in the so-called vibrational fingerprint spectral region [1].

In this work, we demonstrate that Ge antenna structures are also suitable for nonlinear optical processes such as third harmonic generation (THG) in the mid infrared [2], owing to the strong resonant enhancement. Subwavelength-confined light emitters are of high interest for experiments targeting single molecules or other isolated quantum systems [3].
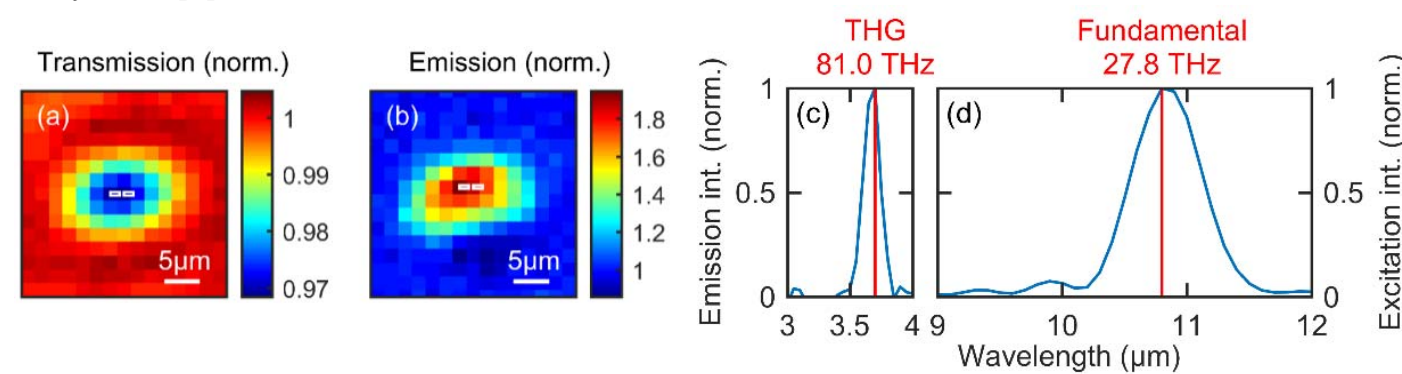

Fig. 1 (a) Linear transmission map of a 2.75- $\mu \mathrm{m}$-long double rod antenna illuminated with a central wavelength of $10.8 \mu \mathrm{m}$ including a sketch of the structure (white); (b) spatially resolved nonlinear emission after 6 - $\mu \mathrm{m}$-short-pass sapphire filter normalized to the silicon substrate background emission; (c) and (d) normalized THG and excitation intensity spectra, respectively.

Doped single-crystalline germanium films are grown via low-energy plasma enhanced chemical vapor deposition (LEPECVD) on intrinsic silicon substrates. Double-rod antenna structures are fabricated subsequently via electron beam lithography to be resonant at the fundamental excitation [4].

The optical system is driven by a $\mathrm{Yb}: \mathrm{KGW}$ femtosecond laser equipped with optical parametric amplifiers. Intense few-cycle pulses tunable in the mid-infrared spectral range are obtained via difference frequency generation in GaSe. Excitation fields of up to $20 \mathrm{MV} / \mathrm{cm}$ are reached in the focus of a Cassegrain-Schwarzschild reflecting objective. A second objective images the antenna in transmission geometry. A mercury cadmium telluride (MCT) detector cooled by liquid nitrogen collects the emission while the sample is scanned through the confocal region. This allows addressing single antennas and mapping their linear and nonlinear response.

Fig. 1(a) shows the transmission image at the fundamental excitation wavelength $(10.8 \mu \mathrm{m}$, Fig. 1(c)) with the extinction due to the increased resonant scattering while Fig. 1(b) plots the corresponding THG emission at $3.7 \mu \mathrm{m}$ wavelength (Fig. 1(d)). The nonlinearity is strongly enhanced at the antenna with respect to the substrate.

In conclusion, semiconductor plasmonic antennas enhance the nonlinear optical emission at the nanoscale with interesting perspectives in coherent ultrafast nearfield microscopy.

This research has received funding from the European Union's Seventh Framework Programme (grant agreement $\mathrm{n}^{\circ}$ 613055) and from the Deutsche Forschungsgemeinschaft (Emmy Noether Program BR 5030/1-1).

\section{References:}

[1] L. Baldassarre, E. Sakat, J. Frigerio, A. Samarelli, K. Gallacher, E. Calandrini, G. Isella, D. J. Paul, M. Ortolani, and P. Biagioni,

"Midinfrared Plasmon-Enhanced Spectroscopy with Germanium Antennas on Silicon Substrates", Nano Lett. 15, 7225-7231 (2015).

[2] N. K. Hon, R. Soref, and B. Jalali, "The third-order nonlinear optical coefficients of Si, Ge, and Sil- x Gex in the midwave and longwave infrared", J. Appl. Phys. 110, 11301 (2011).

[3] T. Hanke, J. Cesar, V. Knittel, A. Trügler, U. Hohenester, A. Leitenstorfer, and R. Bratschitsch, "Tailoring spatiotemporal light confinement in single plasmonic nanoantennas", Nano Lett. 12, 992 (2012).

[4] J. Frigerio, A. Ballabio, G. Isella, E. Sakat, P. Biagioni, M. Bollani, E. Napolitani, C. Manganelli, M. Virgilio, A. Grupp, M. P. Fischer, D. Brida, K. Gallacher, D. J. Paul, L. Baldassarre, P. Calvani, V. Giliberti, A. Nucara, and M. Ortolani, "Tunability and Losses of Mid-infrared Plasmonics in Heavily Doped Germanium Thin Films”, Phys. Rev. B 94, 85202 (2016). 